\title{
Die Sicherheits- und Verteidigungspolitik der Europäischen Union im Praxistest: zwischen Anspruch und Wirklichkeit
}

\author{
Hans-Georg Ehrhart*
}

Das Inkrafttreten des Vertrags von Lissabon ist gerade drei Jahre her, da befindet sich die Europäische Union einmal mehr im Umbruch. Die Krise des Weltfinanzsystems und die Verschuldungskrise scheinen aus Europa eine Großbaustelle gemacht zu haben. Es gibt Katastrophenszenarien über den Untergang der Europäischen Union und den Rückfall Europas in ethnische Konflikte, ${ }^{1}$ aber auch kühne Pläne zur Beschleunigung des Integrationsprozesses und heftige Diskussionen, welcher dieser Pläne den richtigen Weg weist, wie sie umgesetzt werden sollen, ob sie für die Bevölkerung akzeptabel sind und was dafür von wem zu zahlen ist. ${ }^{2}$ Im Grunde genommen geht es seit Beginn der europäischen Integration um die Frage der Finalität: Welches Europa wollen wir haben? Aus der Antwort auf diese interessengeleitete und normative Frage lässt sich ableiten, welche außen- und sicherheitspolitische Rolle die Europäische Union spielen kann. Drei Modelle sind denkbar: 1. Die Vereinigten Staaten von Europa, also eine vollständige Föderation inklusive einer vergemeinschafteten Außen-, Sicherheits- und Verteidigungspolitik. Demnach verläuft der Weg zu einem supranationalen Europa entweder über einen konstitutionellen Akt der Mitglieder (Föderalismus) oder über einen Verflechtungsprozess (Neofunktionalismus). 2. Das Europa der Vaterländer mit einem gemeinsamen Binnenmarkt, dessen Sicherheit durch die Mitgliedstaaten und durch Bündnisse gewährleistet wird. Demnach ist dauerhafte Integration aufgrund der anarchischen Struktur des internationalen Systems nicht möglich (Struktureller Realismus). 3. Das Europa der immer engeren Union der Völker als Gebilde sui generis, das eine Gemeinsame Außen- und Sicherheitspolitik (GASP) und eine Gemeinsame Sicherheits- und Verteidigungspolitik (GSVP) aufbaut, die zu einer gemeinsamen Verteidigungspolitik führt, „sobald der Europäische Rat dies einstimmig beschlossen hat". Demnach ist ein stärker integriertes Europa aufgrund zunehmender Interdependenzen möglich, jedoch reversibel (liberaler Institutionalismus). ${ }^{3}$

Während die beiden ersten Modelle gegenwärtig eher unwahrscheinlich erscheinen, aber im Bereich des Möglichen bleiben, ist das dritte Modell auf absehbare Zeit die realistischere Variante. Mit zunehmender Komplexität fächert es sich auf in flexible Integrationsmuster, die unterschiedliche Integrationsdichten und -geschwindigkeiten umfassen. Das gilt auch für den Bereich der äußeren Sicherheit, obwohl er immer noch eine primär zwischenstaatliche Domäne ist. Der dritte Erklärungsansatz bildet die Interpretationsfolie für diesen Beitrag, der zunächst kurz auf den Wandel der GASP/GSVP eingeht. Anschließend werden die Aktivitäten der Europäischen Union als Krisenmanager in Somalia, Syrien und Mali beleuchtet.

* Dr. Hans-Georg Ehrhart, Mitglied der Geschäftsleitung und Leiter des Zentrums für EUropäische Friedens- und Sicherheitsstudien am Institut für Friedensforschung und Sicherheitspolitik an der Universität Hamburg.

1 Nicholas Sambanis: The euro crisis as ethnic conflict, in: International Herald Tribune, 27.8.2012.

2 Vgl. beispielsweise Guido Westerwelle: Der Wert Europas. Vier Thesen zum Zukunftsprojekt Europa, in: integration 2/2012, S. 90-93; Viviane Reding: Fremde Federn. Mit einer Vision aus der Krise finden, in: Frankfurter Allgemeine Zeitung, 9.3.2012.

3 Vgl. Bernhard Rinke: Die beiden großen deutschen Volksparteien und das „Friedensprojekt Europa“. Weltmacht, Zivilmacht, Friedensmacht?, Baden-Baden 2006, S. 58-78. 
Abschließend folgt eine Erörterung der Perspektiven des Krisenmanagers Europäische Union.

\section{Die Europäische Union im Wandel}

Angesichts einer sich rasant verändernden Welt und neuer Risiken steht die Europäische Union vor der Herausforderung, ihre internationale Rolle zu finden. Seien es die aufstrebenden Mächte der BRICS-Staaten, ${ }^{4}$ die revolutionären Prozesse der ,Arabellion' oder die Verwerfungen im Weltfinanzsystem, diese Prozesse spiegeln Machtverschiebungen wider, zu denen sich die Europäische Union verhalten muss. Einerseits spielt sie eine wichtige Rolle beim Aufbau einer stabilen gesamteuropäischen Friedensordnung. Andererseits strebt sie an, auch global eine mitbestimmende Rolle im Rahmen eines effektiven Multilateralismus zu übernehmen. Die Fähigkeiten dazu sollen ihr die GASP/GSVP verleihen. Deren Werdegang seit den Verträgen von Maastricht, Amsterdam, Nizza und dem gescheiterten Vertrag über eine Verfassung für Europa bis zum Vertrag von Lissabon belegt, dass es sich um einen sehr mühsamen Prozess handelt, der bei Weitem nicht so erfolgreich verlaufen ist wie die währungspolitische Integration, die ja lange Zeit als ausschließlich nationale Domäne angesehen wurde. Gleichwohl brachte jeder Schritt die Union etwas voran. Diese Fortschritte werden deutlicher, wenn man das bislang Erreichte mit der Lage vor dem Vertrag von Maastricht, also mit der Europäischen Politischen Zusammenarbeit vergleicht. Gemessen an den Herausforderungen des 21 . Jahrhunderts gibt es aber noch viel zu tun, um die angestrebte internationale Rolle übernehmen zu können. ${ }^{5}$

Ist die Einigung auf einen Vertragstext bereits schwer genug, wie die Vorlaufzeit von acht Jahren seit dem Startschuss des Reformprozesses durch den Europäischen Rat in Laeken über den gescheiterten Verfassungsvertrag bis zur Ratifizierung zeigt, so stellt dessen Implementierung in der politischen Praxis eine noch größere Herausforderung dar. Dementsprechend warnen Praktiker und Experten zu Recht vor zu großen Erwartungen gerade im Bereich der GASP. So spricht Helga-Maria Schmid von einer „Übergangszeit“ und „vom Anfang des Prozesses, an dessen Ende eine effektive, effiziente und überzeugende EU-Außen- und Sicherheitspolitik stehen wird“. ${ }^{6}$ Andere beschreiben den Übergang zu einem neuen außenpolitischen System nach ,Lissabon“ als ,,work in progress“", 7 als fortlaufenden Prozess, der noch viel politisches Engagement erfordere. Zwar wurden mit dem Europäischen Auswärtigen Dienst (EAD) ein neuer institutioneller Rahmen und mit den erweiterten Kompetenzen der Hohen Vertreterin der Union für Außen- und Sicherheitspolitik (HV/VP) neue Handlungsmöglichkeiten geschaffen, doch sind diese bislang noch bei Weitem nicht ausgeschöpft worden.

Es ist insbesondere die enge Verbindung verschiedener Politikfelder und damit die Option auf eine umfassende Strategie, die durch die Reformen des Vertrags von Lissabon ermöglicht werden sollen. Es geht darum, den oft beschworenen Mehrwert der Europäischen Union, also die Vielzahl der Instrumente der Union und ihrer Mitgliedstaaten, besser für die GASPPraxis nutzbar zu machen. Insofern eröffnet das europäische Primärrecht in der Tat eine

4 Brasilien, Russland, Indien, China und Südafrika.

5 Thomas Renard/Sven Biscop (Hrsg.): The European Union and Emerging Powers in the $21^{\text {st }}$ Century. How Europe Can Shape a New Global Order, Farnham 2012.

6 Helga-Maria Schmid: EU-Außenpolitik nach Lissabon. Struktur und Wirkung, in: Zeitschrift für Außen- und Sicherheitspolitik 4/2010, S. 457-465, hier S. 458.

7 Antonio Missiroli: The New EU 'Foreign Policy’ System after Lisbon: A Work in Progress, in: European Foreign Affairs Review 4/2010, S. 427-452, hier S. 429. 
Möglichkeit „to generate not only better coordination and coherence, but even synergy between all the different aspects of the Union's external action". ${ }^{8}$ Letztlich geht es darum, einen vernetzten beziehungsweise ganzheitlichen Ansatz - im mittlerweile von der Europäischen Union übernommenen NATO-Jargon ${ }^{9}$ spricht man von einem , comprehensive approach ${ }^{\text {- }}$ zu ermöglichen, der die Trennungen überwindet und Synergien schafft: zwischen den Instrumenten und Politiken der Sicherheits- und Entwicklungspolitik, der inneren und äußeren Sicherheit, der Diplomatie und dem Krisenmanagement sowie dem zivilen und militärischen Bereich. ${ }^{10}$ Ad-hoc-Einrichtungen im EAD, wie das Crisis Management Board oder die Crisis Platform, sind erste Versuche, diesen Ansatz umzusetzen. ${ }^{11}$ Gleichwohl klaffen Anspruch und Wirklichkeit bei konkreten Engagements in Konflikten auseinander.

\section{Europäische Union als Krisenmanager}

Nach der Unterzeichnung des Vertrags von Lissabon engagierte sich die Europäische Union in drei Krisen mit unterschiedlicher Intensität und verschiedenen Mitteln. ${ }^{12}$ In Somalia ergänzte sie ihre politischen, entwicklungspolitischen und humanitären Aktivitäten durch zivile und militärische Maßnahmen zur Bekämpfung der Piraterie. In Syrien beschränkte sie sich auf diplomatische und humanitäre Maßnahmen, während einzelne Mitgliedstaaten diplomatisch vorpreschten und auch militärisch aktiv wurden. In Mali wiederum startete die Europäische Union eine neue zivile GSVP-Operation, überließ das militärische Eingreifen aber Frankreich und afrikanischen Partnern. ${ }^{13}$

\section{Somalia}

Die Europäische Union engagiert sich in Somalia seit Beginn des letzten Jahrzehnts nicht nur humanitär. Angesichts der Anschläge vom 11. September 2001 und der dadurch gestiegenen Bedeutung von sogenannten gescheiterten Staaten in der internationalen Bedrohungswahrnehmung setzte sich ,Brüssel' zunächst mit entwicklungspolitischen und diplomatischen, später auch mit polizeilichen und militärischen Instrumenten dafür ein, Somalia zu stabilisieren. Dabei verfolgt die europäische Politik nach eigenem Bekunden einen ,com-

8 Ebenda, S. 445.

9 North Atlantic Treaty Organization.

10 Vgl. Rat der Europäischen Union: Military Advice on the further handling of the Comprehensive Approach to EU Crisis Management, Brüssel, 30. März 2010; Isabelle Tannous: Der Europäische Auswärtige Dienst und die Organisation europäischer Außen- und Entwicklungshilfe: von institutionellen Dissonanzen zur dienstübergreifenden Harmonie?, in: integration 4/2012, S. 274-295.

11 Vgl. Linda Barry: European Security in the $21^{\text {st }}$ Century: The EU's Comprehensive Approach, Dublin 2012, S. 8.

12 Seit 2009 begann die Europäische Union zudem die Missionen EUCAP Sahel-Niger und EUAVSEC South Sudan. Die für eine humanitäre Mission während der militärischen Auseinandersetzung in Libyen vorgesehene EUFOR Libya kam nicht zum Einsatz. Dafür gab die Europäische Union Anfang 2013 grünes Licht für die Grenzsicherungsmission EUBAM Libya. Vgl. die Übersicht über alle EU-Operationen European Union External Action: EU Operations, abrufbar unter: http://ue.eu.int/eeas/security-defence/eu-operations?lang=en (letzter Zugriff: 15.5.2013).

13 Vgl. zur EU-Politik im Libyen-Konflikt Daniel Göler: Die Europäische Union in der Libyen-Krise. Die ,responsibility to protect' als Herausforderung für die strategischen Kulturen in Europa, in: integration 1/2012, S. 3-18. 
prehensive approach', der insbesondere auf die Stärkung der somalischen Staatlichkeit, Piratenbekämpfung und den Aufbau von sicherheitspolitischen Kapazitäten setzt. ${ }^{14}$

Die Europäische Union ist einer der größten Geber von humanitärer Hilfe (49 Millionen Euro in 2012) und von Entwicklungshilfe (412 Millionen Euro im Zeitraum von 2008 bis 2013). Geförderte Kernfelder sind Regierungsführung, Sicherheit, Bildung, wirtschaftliche Entwicklung und Nahrungsmittelsicherheit. Die Europäische Union setzte sich zusammen mit den Nachbarstaaten, regionalen Organisationen und den Vereinten Nationen für einen Friedensprozess ein, der schließlich 2004 zur Bildung einer Übergangsregierung (Transitional Federal Government, TFG) führte. Diese blieb bis zum Ende der Transitionsphase im September 2012 der Hauptansprechpartner und Hauptempfänger von Leistungen - obwohl sie als korrupt und unzuverlässig eingeschätzt wurde. ${ }^{15}$ Die neue Regierung hat nun die Aufgabe, die Gewalt in Süd-Zentral-Somalia zu beenden und die neuen Institutionen vom Volk bestätigen zu lassen. Die 2011 beschlossene Strategie für das Horn von Afrika nennt folgende Handlungsschwerpunkte: demokratischer Staatsaufbau, Frieden, Sicherheit, regionale Zusammenarbeit, Armutsbekämpfung und Wirtschaftsförderung. Die Lage in Somalia und die Piraterie werden als Hauptprobleme identifiziert und stehen im Mittelpunkt der Arbeit des 2012 ernannten EU-Sonderbeauftragten für die Region. ${ }^{16}$

$\mathrm{Ab} 2007$ setzte die Union zunehmend auf militärische Mittel. Zunächst unterstützte sie die Friedenstruppe der Afrikanischen Union (AMISOM) mit insgesamt 407 Millionen Euro aus dem Europäischen Entwicklungsfonds bei der Aufgabe, die Übergangsregierung zu sichern und humanitäre Hilfe zu ermöglichen. AMISOM engagierte sich seit 2011 verstärkt in der Aufstandsbekämpfung gegen die radikalislamische al-Shabaab und wurde dabei von Kenia (das seine Interventionskräfte später der AMISOM eingliederte) und Äthiopien sowie gelegentlichen punktuellen Einsätzen von Spezialkräften der USA, Frankreichs und Großbritanniens unterstützt. ${ }^{17}$

2008 startete mit „EU Naval Force Somalia - Operation Atalanta“ die erste Marineoperation im Rahmen der GSVP. Basierend auf einer Anforderung der somalischen Regierung und der Vereinten Nationen von Unterstützung im Kampf gegen Piraterie legte der Rat der Europäischen Union vier Ziele fest, zu deren Erreichung die Mission beitragen soll: 1. Schutz der Schiffe des Welternährungsprogramms (World Food Programme, WPF) und von AMISOM-Transporten; 2. Schutz verwundbarer Schiffe; 3. Prävention, Abschreckung und Repression von Piraterie; und 4. Überwachung von Fischereiaktivitäten vor der somalischen Küste. Das übergreifende politische Ziel lautet „to improve maritime security in the region“, das politisch-militärische ,to deter piracy and to strengthen the security of main maritime routes". ${ }^{18}$ Wohl wissend, dass eine völlige Ausschaltung der Piraterie unmöglich ist, spricht

14 Vgl. zum Folgenden Hans-Georg Ehrhart/Kerstin Petretto: The EU and Somalia. Counter-Piracy and the Question of a Comprehensive Approach, Study for The Greens/European Free Alliance, Hamburg 2012; Hans-Georg Ehrhart/Kerstin Petretto: The EU, the Somalia challenge, and counter-piracy. Towards a comprehensive approach?, in: European Foreign Affairs Review 2/2012, S. 261-283.

15 Jason Straziuso: UN report cites massive corruption in Somali government, in: Bloomberg Businessweek, 16.7.2012, abrufbar unter: http://www.businessweek.com/ap/2012-07-16/un-report-cites-massive-corruptionin-somali-govt (letzter Zugriff: 17.5.2013).

16 Rat der Europäischen Union: Horn von Afrika - Schlussfolgerungen des Rates, Dok. 16858/11.

17 The Bureau of Investigative Journalism: Covert War on Terror - the Data. Somalia. Reported US covert actions 2001-2012, 22.2.2012; Vereinte Nationen: Report of the Monitoring Group on Somalia and Eritrea pursuant to Security Council resolution 2002 (2011), S/2012/544.

18 Rat der Europäischen Union: Projet de concept de gestion de crise (CMC) pour une action de l'Union Européenne en vue d'une contribution à la mise en œuvre de la résolution 1816 (2008) du Conseil de sécurité des Nations Unies, Dok. 12335/08, S. 12-13. 
die Europäische Union vom Ziel ihrer dauerhaften Reduzierung, die nur durch ein angemessenes Niveau von Recht und Ordnung, Entwicklung und Sicherheit erreicht werden könne. ${ }^{19}$

Zwei Jahre später engagierte sich die Europäische Union mit einer Ausbildungsmission für somalische Sicherheitskräfte an Land. Die „European Union military mission to contribute to the training of Somali security forces" (EUTM Somalia) ist ein Beitrag zur Reform des Sicherheitssektors durch die Ausbildung von 3.000 Rekruten für die Nationalen Sicherheitskräfte Somalias. Sie findet in Uganda statt und ist Teil eines internationalen Projekts, das in enger Koordination mit den Vereinten Nationen, AMISOM, den USA und Uganda durchgeführt wird. Die Europäische Union erwägt eine spätere Verlegung nach Somalia, falls sich die dortige Lage stabilisieren sollte. ${ }^{20}$ Eine weitere GSVP-Mission startete 2012 mit dem Namen „Regional Maritime Capacity Building in the Horn of Africa“ (EUCAP NESTOR). Dabei handelt es sich um eine zivile Ausbildungsmission zur Stärkung der maritimen Fähigkeiten ausgewählter Staaten in der Region und in Somalia selbst. ${ }^{21}$

Eine Bewertung des bisherigen EU-Engagements fällt gemischt aus. Bezüglich der internen Abstimmung kann festgestellt werden, dass der EAD sein Potenzial nur bedingt zur Geltung bringen konnte. Das liegt zum einen daran, dass er erst spät arbeitsfähig wurde; zum anderen ist der proklamierte umfassende Ansatz nicht wirklich integrativ. Was generell immer noch fehlt, ist eine integrierte strategische Planung. Immerhin werden die drei GSVPMissionen mittlerweile vom aktivierten operativen Hauptquartier in Brüssel aus geleitet oder koordiniert. ${ }^{22}$ Die Abstimmung mit anderen Akteuren wie internationalen und regionalen Organisationen sowie staatlichen und nichtstaatlichen Akteuren ist sehr intensiv. Gleichwohl setzte die dringend benötigte humanitäre Hilfe während der Hungerskatastrophe $2011 \mathrm{zu}$ spät ein und erreichte die Adressaten in Somalia unzureichend. Den Schutz der Schiffe des WFP und der AMISOM-Transporte gewährleistete Atalanta allerdings zu 100 Prozent, den von Handelsschiffen nur zum Teil. Die Erfolgsrate der Piraten konnte, trotz bis Mitte 2011 gestiegener Angriffszahlen, reduziert werden. Die Ausdehnung des Aktionsradius der Piraten erwies sich bis dahin aber als Problem. Sie konnten auch erhöhte Lösegeldforderungen durchsetzen, sodass sie obwohl weniger Schiffe gekapert wurden mehr Geld einnahmen. ${ }^{23}$

Die Tatsache, dass die Angriffszahlen 2012 stark gesunken sind kann nicht darüber hinwegtäuschen, dass der Auftrag von Atalanta nur partiell erfüllt werden konnte. ${ }^{24}$ Die Piraten sind weiterhin aktiv, wenn auch auf sehr niedrigem Niveau, und das Monitoring der Fischereiaktivitäten wurde erst gar nicht ernsthaft umgesetzt. Die Gründe dafür liegen darin, dass der Auftrag gemessen an den zur Verfügung gestellten Ressourcen - fünf bis zehn Schiffe und maximal vier Seeaufklärer für den ganzen Indischen Ozean - zu anspruchsvoll ist und dass vor allem zu wenig unternommen wurde, um die Ursachen des Übels an Land zu bearbeiten. So wurden die meisten der gefassten Piraten wieder freigelassen, weil es vor Ort

19 Ebenda, S. 14.

20 Europäischer Auswärtiger Dienst: EUTM Somalia, abrufbar unter: http://ue.eu.int/eeas/security-defence/eu-op erations/eu-somalia-training-mission?lang=en (letzter Zugriff: 7.2.2013).

21 Europäischer Auswärtiger Dienst: EUCAP Nestor, abrufbar unter: http://ue.eu.int/eeas/security-defence/eu-op erations/eucap-nestor?lang=en (letzter Zugriff: 7.2.2013).

22 Das OpsCent übernimmt je nach Operation unterschiedliche Führungsaufgaben. Vgl. Nicolas Gros-Verheyde: L'OpsCenter sur les rails: les grands principes adoptés lundi. Et quelques détails..., Buxelles2.eu, 20.1.2012.

23 Vgl. Ehrhart/Petretto: The EU, the Somalia Challenge and Counter Piracy, 2012, S. 275.

24 Folgende Gründe können als Ursache der verbesserten Lage angenommen werden: optimierte Präsenz der internationalen Flotten, Erweiterung des Mandats um gezielte Angriffe auf die Infrastruktur der Piraten an Land, verbesserte Umsetzung der ,best management practices` seitens der Reeder, Einsatz privater Sicherheitsdienste auf den Schiffen. 
kein funktionierendes Justizwesen gibt und viele EU-Mitgliedstaaten angesichts der schweren Beweislage, der relativ hohen Kosten und der mangelnden direkten Betroffenheit davor zurückschrecken, die Piraten in der Europäischen Union vor Gericht zu stellen. ${ }^{25}$

Die Ausbildung somalischer Sicherheitskräfte durch die EUTM Somalia und andere dient der Unterstützung der somalischen Zentralregierung und dem Kampf gegen die al-Shabaab, aber nicht der Pirateriebekämpfung. Die Arbeit der EUTM Somalia kann durchaus als Erfolg angesehen werden, doch hatte sie Probleme bei der Aufsicht über die ausgebildeten Sicherheitskräfte und deren Führung durch eine zutiefst unpopuläre Übergangsregierung. Das erste Problem lässt sich lösen, wenn die EUTM Somalia wie geplant nach Somalia verlegt wird; die Lösung des zweiten hängt davon ab, wie sich die neuen somalischen Institutionen entwickeln.

Ob EUCAP Nestor mit ihrem Auftrag, die maritimen Fähigkeiten Somalias und der Nachbarstaaten zu verbessern, Erfolg haben wird, bleibt abzuwarten. Diese zunächst für zwei Jahre mandatierte GSVP-Mission braucht Zeit, um wirken zu können. Sie könnte dazu beitragen, den Aufbau einer Küstenpolizei und der Justiz zu unterstützen, wobei sie sich zunächst auf Puntland und Somaliland konzentrieren wird, weil es dort leidlich funktionierende lokale Administrationen gibt. Sie setzt also in einem Sektor an, der von der internationalen Gemeinschaft bislang sträflich vernachlässigt worden ist. Zugleich weicht sie von der gängigen aber kritikwürdigen Praxis ab, sich primär auf die Zentralregierung zu konzentrieren.

Grundsätzlich stellt sich allerdings die Frage, wie sich die politische Landschaft Somalias nach über 20 Jahren ohne Zentralregierung und dem Ende der achtjährigen Transitionsphase entwickelt. Das Ende der Transition ist ein wichtiger Zwischenschritt, zu dem auch die Europäische Union mit ihren diplomatischen, entwicklungspolitischen, humanitären und GSVP-Instrumenten beigetragen hat. Nun müssen die neuen politischen Institutionen funktionieren und sich demokratisch legitimieren lassen. Angesichts der politischen Zersplitterung des Landes in verschiedene Entitäten, der komplexen Sozialstruktur mit ihrem ausdifferenzierten Clansystem und den massiven humanitären und entwicklungspolitischen Herausforderungen müsste sich die Europäische Union zusammen mit ihren internationalen Partnern auf ein langfristiges Engagement einstellen, wenn sie denn wirklich die Ursachen von Piraterie an Land beseitigen will. Die Frage ist, ob sie zu einem nachhaltigen Engagement bereit ist.

\section{Syrien}

Die Syrienpolitik der Europäischen Union wurde vor dem Ausbruch der Krise, die dann in einen Bürgerkrieg mündete, geleitet durch institutionalisierte bi- und multilaterale Beziehungen wie etwa dem Kooperationsabkommen von 1977, der Erklärung von Barcelona aus dem Jahr 1995, der Mitgliedschaft Syriens in der 2008 beschlossenen Union für das Mittelmeer und der Europäischen Nachbarschaftspolitik. Die im März 2011 einsetzende Unterdrückung der syrischen Protestbewegung durch die Regierung des syrischen Präsidenten Bashar al-Assad führte zu einer sich zunehmend verschärfenden Konfliktlage, die auf die ganze Region ausstrahlt. Die von ,Brüssel' zur Krisenbearbeitung eingesetzten Mittel umfassen humanitäre Hilfe, Diplomatie und Sanktionen. Im humanitären Bereich erfordern die

25 Einerseits ist Piraterie ein universelles Delikt, das jeder Staat verfolgen kann, andererseits sind die meisten Staaten, wenn überhaupt, dazu nur bereit, wenn eine eindeutige Verbindung zwischen dem jeweiligen Staat und dem Angriff besteht. 
drastisch steigenden Flüchtlingszahlen entsprechendes internationales Engagement. Die Europäische Union und die Mitgliedstaaten stellten bis zum 11. Mai 2013 Hilfsgelder in Höhe von 626 Millionen Euro zur Verfügung. ${ }^{26}$

Zudem erließ die Europäische Union Schritt für Schritt mehr Sanktionen als jemals zuvor. Die insgesamt 22 Sanktionen umfassen gezielt Maßnahmen gegen die Regierung (zum Beispiel Stopp der Leistungen der Europäischen Investitionsbank), gegen an der Repression beteiligte Individuen (zum Beispiel durch die Erstellung einer schwarzen Liste von unerwünschten Personen) und gegen staatliche Institutionen (zum Beispiel Waffenembargo), gegen den Energiesektor (zum Beispiel Ölembargo) und gegen die Wirtschaft (zum Beispiel Exportverbote für bestimmte Güter). Darüber hinaus haben einzelne Mitgliedstaaten ihre Botschaften in Syrien geschlossen. ${ }^{27}$ Dieser massive Druck durch umfassende Sanktionen ist ungewöhnlich für die Europäische Union, hat sie doch bislang gerade in der Nahostregion eher zurückhaltend mit diesem Instrument agiert und treffen die Sanktionen doch auch einzelne EU-Staaten. Allerdings dürften sich Wirkungen erst mittel- und langfristig zeigen. Ein Problem besteht darin, dass die Sanktionen zwar parallel zu den US-Sanktionen laufen und von der Arabischen Liga (AL) unterstützt werden, aber eben doch nicht von allen Mitgliedern im Sicherheitsrat der Vereinten Nationen mitgetragen werden. Ein anderes Problem ist, dass die Palette der nicht-militärischen Sanktionen jetzt weitgehend ausgeschöpft ist.

Anfangs zielte die EU-Politik darauf ab, Assad zu Verhandlungen mit der Opposition zu bewegen, seine Regierung von einer gewaltsamen Lösung abzuhalten und dabei Unterstützung seitens der internationalen Partner zu gewinnen. Mit Zuspitzung der Lage setzte die Europäische Union vor allem auf die AL und die Vereinten Nationen. So unterstützte sie die Beobachtermission der AL mit diplomatischen und technischen Mitteln. Sie machte sich auch die Forderung der AL zu eigen, dass Assad die Macht abgeben solle. Als Mitglied der von Frankreich initiierten Gruppe „Friends of the Syrian People“28 trägt sie zur Abstimmung der internationalen Aktivitäten bei. Der Widerstand Chinas und Russlands im Sicherheitsrat der Vereinten Nationen gegen eine aus ihrer Sicht zu intrusive Politik der Europäischen Union und der USA schränkt aber nicht nur die Wirksamkeit der Sanktionen ein, sondern zeigt auch, dass die Partnerschaft der Europäischen Union mit diesen Staaten in diesem konkreten Fall bislang noch keine positive Wirkung entfaltet. Deshalb üben die EU-Staaten über die Bühnen der Vereinten Nationen wie dem Sicherheitsrat, dem Menschenrechtsrat und der Vollversammlung diplomatischen Druck auf beide Staaten aus, um sie dazu zu drängen, ihr Verhalten zu rechtfertigen.

Einerseits ist die Feststellung zutreffend, dass „Syria has become a high-profile test of the Union's capabilities“. ${ }^{29}$ Da bislang in der Europäischen Union und den USA weitgehend Konsens darüber besteht, dass die offene Anwendung militärischer Zwangsmaßnahmen nicht wünschenswert ist, kommt nicht-militärischen Ansätzen und Instrumenten die zentrale Rolle zu. Andererseits stellt sich die Frage, wie lange diese Beschränkung aufrechterhalten wird und wie sich die EU-Mitgliedstaaten hinsichtlich einer möglichen Aufweichung posi-

26 Europäische Kommission: Echo Fact Sheet Syria, 11.5.2013, abrufbar unter: http://ec.europa.eu/echo/files/aid /countries/factsheets/syria en.pdf (letzter Zugriff: 18.5.2013).

27 Clara Portela: The EU's Sanctions against Syria. Conflict Management by Other Means, Egmont: Security Policy Brief 38/2012; Liste der Sanktionen im Annex von: European Union: Factsheet. The European Union and Syria, 22.4.2013, abrufbar unter: http://www.consilium.europa.eu/uedocs/cms_data/docs/pressdata/EN/for aff/128379.pdf (letzter Zugriff: 18.5.2013).

28 An den bisherigen Treffen nahmen fast 90 Staaten, sechs internationale Organisationen und der syrische Nationalrat als Dachorganisation der Oppositionsgruppen teil.

29 Richard Gowan: The EU and Syria: everything but force?, Opinion, Institute for Security Studies, 26.1.2012. 
tionieren. Catherine Ashton wurde vor dem Europäischen Parlament ziemlich deutlich, als sie eine gefährliche Pattsituation konstatierte sowie die Notwendigkeit ,to do everything we possibly can to find ways to promote a political transition. [...] Our approach, in a sense, must change; we have to make real progress on this“. ${ }^{30}$

Die Frage ist nur, worin dieser Positionswandel bestehen soll. Nach den Erfahrungen mit der Überdehnung des Mandats der Resolution 1973 des Sicherheitsrates der Vereinten Nationen in Libyen stemmen sich Russland und China energisch gegen eine Politik des von außen geförderten Regimewechsels. Gleichwohl haben Staaten aus der Region, die USA und auch EU-Mitglieder erste militärisch relevante Schritte unternommen: Die Türkei stellt ihr Gebiet als Rückzugsraum und für Ausbildungszwecke zur Verfügung und liefert sich mit syrischen Streitkräften Grenzscharmützel; Katar und Saudi-Arabien finanzieren und liefern Waffen, die USA, Frankreich und Großbritannien helfen mit Ausbildung, Kommunikationsmitteln und Aufklärung, wobei Deutschland vom Mittelmeer aus durch ein Spezialschiff mit Radaraufklärung beiträgt. ${ }^{31}$ Zudem ist anzunehmen, dass die NATO Ergebnisse weiterleitet, die sie aus der im Rahmen der „Operation Active Endeavour“ erfolgten Seeraumüberwachung des östlichen Mittelmeers gewinnt. ${ }^{32}$

Bei einer weiteren Verschärfung der Lage für die Zivilbevölkerung könnte die Einrichtung humanitärer Zonen und einer Flugverbotszone erwogen werden. Allerdings würde das ohne Mandat der Vereinten Nationen eine direkte militärische Intervention mit unvorhersehbarem Eskalationspotenzial bedeuten - ein Risiko, das Washington, Paris und London bislang noch scheuen. ${ }^{33}$ Gleichwohl lockerte die Europäische Union auf Drängen Frankreichs und Großbritanniens das Embargo etwas, indem sie etwa die Lieferung nichttödlicher Ausrüstung wie Schutzwesten und Nachtsichtgeräte erlaubte. ${ }^{34}$ Frankreich hat als erstes Land die heterogene Nationale Koalition der syrischen Opposition als ,die einzige legitime Vertretung des syrischen Volkes“" anerkannt, ${ }^{35}$ während sich die EU-Außenminister zunächst auf den Kompromiss einigten, sie bloß als ,legitimate representative of the Syrian people“ anzuerkennen. ${ }^{36}$ Der Schulterschluss gelang dann am 20. April 2013. ${ }^{37}$ Der Beschluss der NATO, Luftabwehrbatterien vom Typ Patriot 3 in der Türkei zu stationieren, um diese vor einem syrischen Angriff zu schützen, wird zwar als reine Abschreckungsmaßnahme begründet, erhöht aber den militärischen Druck auf Damaskus, weil diese Raketen neue militärische Optionen ermöglichen könnten.

30 Catherine Ashton: Statement by EU High Representative Catherine Ashton in the European Parliament on the situation in Syria, A 404/12, Straßburg 11.9.2012, S. 2.

31 Nicolas Gros-Verheyde: Français et Britanniques engagés aux côtés des insurgés, in: Bruxelles2.eu, 24.8.2012.

32 Thomas Wiegold: Spionageschiff? Spionageflugzeug!, in: Augen geradeaus!, 23.8.2012.

33 Vgl. zu den möglichen militärischen Optionen Markus Kaim: Crisis in Syria. Possibilities and Limits of Military Intervention, Stiftung Wissenschaft und Politik: SWP Comments 11/2012.

34 FAZ.net: EU verlängert Waffenembargo, 28.2.2013.

35 Steven Erlanger: In a boost for Syrian rebels, France to offer recognition, in: International Herald Tribune, 14.11.2012.

36 Rat der Europäischen Union: $3209^{\text {th }}$ Council meeting. Foreign Affairs, Dok. 17438/12, S. 10.

37 Auswärtiges Amt: Joint Statement of the Participating Countries in the Istanbul Meeting on Syria, 20.5.2013, abrufbar unter: http://www.auswaertigesamt.de/EN/Aussenpolitik/Laender/Aktuelle_Artikel/Syrien/130420_I stanbul_TreffenFreundesgruppe.html (letzter Zugriff: 18.5.2013). 
Während sich die Konfliktparteien innerhalb Syriens mit aller Härte bekämpfen, steigt die Zahl der Toten, Verwundeten und Flüchtlinge. ${ }^{38} \mathrm{Im}$ Grunde genommen droht nach fast zwei Jahren Bürgerkrieg das schlimmste aller Szenarien: Das ganze Land ist Schauplatz von Auseinandersetzungen, keine Partei ist in der Lage zu gewinnen, in den Reihen der Aufständischen kämpfen radikalislamistische Kräfte, die al-Qaida nahe stehen, es gibt erste bewaffnete Auseinandersetzungen zwischen den oppositionellen Gruppen, also einen ,Krieg im Krieg“, und eine horizontale Eskalation des Konflikts in die Nachbarstaaten, welche die ganze Region in Brand setzen könnte. ${ }^{39}$ Die politischen, humanitären und wirtschaftlichen Belastungen für die Nachbarstaaten Türkei, Libanon, Jordanien und Irak nehmen dramatisch zu. Die mehr oder weniger direkte Unterstützung der syrischen Konfliktparteien durch die AL und den Westen einerseits und den Iran und Russland andererseits macht die regionale und globale Brisanz des Konflikts deutlich. Es geht nicht mehr nur um Syrien, sondern auch um die Kurdenfrage, die Stabilität des Libanons und Jordaniens, das saudisch-iranische Ringen um regionale Vorherrschaft, den Palästinakonflikt und nicht zuletzt um den iranisch-israelischen Nuklearkonflikt.

Die EU-Mitglieder sind angesichts dieser Lage uneins und ratlos. Sie sind sich uneinig über die Aufhebung des Waffenembargos und ratlos, wie der Konflikt eingedämmt werden kann. Die Folgen des Libyenkonflikts haben gezeigt, welche destabilisierende Wirkung die unkontrollierte Verbreitung von Waffen für eine ganze Region haben kann. Darum sind Deutschland und die meisten anderen EU-Staaten gegen eine Aufhebung des Waffenembargos, während Frankreich und Großbritannien dafür sind. Neue politische Initiativen wie die von den USA und Russland geplante internationale Syrienkonferenz machen etwas Hoffnung. Dabei könnte die Europäische Union über die GASP vielleicht eine unterstützende Rolle spielen, etwa durch flankierende politische Initiativen, die auf die Erfahrungen aus dem Bosnienkonflikt zurückgreifen. ${ }^{40}$ Die Ernennung eines Sonderbeauftragten für Syrien wäre ein erster kleiner Schritt. Jedoch scheint ihr dazu der politische Wille zu fehlen.

\section{Mali}

Die Politik der Europäischen Union gegenüber Mali war in der Vergangenheit hauptsächlich Entwicklungspolitik. Die wichtigsten Themenfelder der Mali-Srategie 2008-2013 sind Regierungsführung, Entwicklung und Budgethilfe. Doch zeichnet sich eine Tendenz zu einer engeren Verbindung von Entwicklungs- und Sicherheitspolitik ab. Zehn Prozent der im Europäischen Entwicklungsfonds vorgesehenen Mittel entfallen auf die „Strategie für Sicherheit und Entwicklung im Sahel““. ${ }^{41}$ Mali kann nicht losgelöst von der Lage im Sahel gesehen werden, einer der ärmsten Regionen in der Welt, die unter Nahrungsmittelkrisen, schnellem Bevölkerungswachstum, schwacher Staatlichkeit, Korruption, gesellschaftlichen Spannungen, Extremismus sowie Radikalisierung, Schmuggel und Terrorismus leidet.

38 Die Zahl der Flüchtlinge stieg bis Mitte Mai 2013 auf über 1,5 Millionen. Die Zahl der Toten wird auf 94.000 geschätzt. Vgl. Flüchtlingshilfswerk der Vereinten Nationen, UNHCR: Syria Regional Refugee Response, abrufbar unter: http://data.unhcr.org/syrianrefugees/regional.php (letzter Zugriff: 17.5.2013); Reliefweb: 94.000 dead in Syria conflic: NGO revised toll, 14.5.2013, abrufbar unter: http://reliefweb.int/report/syrian-arab-repu blic/94000-dead-syria-conflict-ngo-revised-toll (letzter Zugriff: 17.5.2013).

39 Die israelischen Luftangriffe auf einen Konvoi der Hisbollah in Syrien am 30. Januar 2013 und auf eine militärische Forschungseinrichtung bei Damaskus Anfang Mai sind erste Indizien für eine horizontale Eskalation. Vgl. Peter Münch: Luftangriffe aus Israel. Syrische Regierung und Hisbollah drohen mit Vergeltung, in: Süddeutsche Zeitung, 5.5.2013.

40 Vgl. Christopher Hill: When to talk to monsters, in: International Herald Tribune, 17.5.2013.

41 Europäische Kommission: Mali, abrufbar unter: http://ec.europa.eu/europeaid/where/acp/country-cooperation/ mali/mali_en.htm (letzter Zugriff: 7.2.2013). 
Die Europäische Union verfolgt gegenüber dieser Region seit 2011 eine Strategie, die auf vier Prämissen beruht: 1. Sicherheit und Entwicklung sind untrennbar miteinander verbunden; 2. beides erfordert eine engere regionale Zusammenarbeit; 3. allen Staaten wird ein verstärkter Kapazitätsaufbau in den Bereichen Regierungsführung und Entwicklungszusammenarbeit nützen; 4. die Europäische Union muss dabei eine wichtige Rolle spielen. ${ }^{42} \mathrm{Sie}$ soll den Rahmen für einen umfassenden Ansatz bieten, der alle zur Verfügung stehenden Instrumente umfasst. Eine Taskforce Sahel, der unter anderem der Anti-Terrorismus-Koordinator, Regionalspezialisten des EAD und Militärs angehören, soll die Strategie laufend bewerten und Anpassungsvorschläge machen. Die Umsetzung der Sahel-Strategie ist gegenwärtig auf Mauretanien, Niger und Mali beschränkt. Diese Staaten erhalten 660 Millionen Euro hauptsächlich aus dem Europäischen Entwicklungsfond. ${ }^{43}$ Die Europäische Union hat weitere 167 Millionen Euro im Rahmen ihrer Sahel-Strategie bereitgestellt, die Europäische Kommission angesichts der Flüchtlingswelle zusätzlich 172 Millionen Euro für humanitäre Hilfe. ${ }^{44}$

Im Rahmen der GSVP startete Brüssel im Juli 2012 die zivile Operation „EUCAP SAHEL Niger“. ${ }^{45}$ Sie dient dem Ziel der Bekämpfung des Terrorismus und der organisierten Kriminalität durch Beratung und Unterstützung in den Bereichen Stärkung der Rechtsstaatlichkeit, Sicherheitssektorreform und regionale Zusammenarbeit. 49 Polizei- und Militärexperten aus zehn EU-Staaten arbeiten in der Mission. Zudem stellt die Kommission über ihr Stabilitätsinstrument (IfS) Mittel zur Krisenbearbeitung zur Verfügung. Finanzbeiträge für das „Sonderprogramm für Frieden, Sicherheit und Entwicklung" gehören ebenso dazu wie ein Projekt zur Konfliktminderung im Norden Nigers und das 2011 begonnene Antiterrorismus-Projekt „Strategy for Security and Development in the Sahel (CT Sahel)“, das die Polizeibehörden in Mali, Niger und Mauretanien unterstützen soll. ${ }^{46}$ Darüber hinaus erhält Mali bilaterale sicherheitspolitische Hilfe: So unterstützt Frankreich die malischen Streitkräfte seit Jahren. Deutschland engagiert sich seit 2009 bei der Ausrüstung und Ausbildung eines malischen Pionierbataillons, das für die Soforteinsatzkräfte der Afrikanischen Wirtschaftsgemeinschaft ECOWAS vorgesehen ist. ${ }^{47}$

Nach Einschätzung der Europäischen Union ist die Krise in Mali eine Bedrohung für das Land selbst, die Region und Europa. Die Auswirkungen der Krise auf die wirtschaftliche Situation würden den fragilen politischen Transitionsprozess untergraben und die Stellung der aufständischen und extremistischen Gruppen stärken. Eine dauerhafte Präsenz der Dschihadisten und Kriminellen könnte die Bevölkerung radikalisieren und auf die ganze Region ausstrahlen. Die Europäische Union sieht hier strategische Interessen bedroht: durch Geiselnahmen von EU-Bürgern ${ }^{48}$, die Infiltration der Diasporas durch terroristische Netzwerke, eine Unterbrechung der Uranlieferungen ${ }^{49}$ und die Intensivierung des Drogenschmuggels. ${ }^{50}$

Als erste Reaktion auf den Putsch gegen den Malischen Präsidenten Amadou T. Touré im März 2012 stoppte die Europäische Union die Entwicklungszusammenarbeit. Sie begrüßte

42 Europäischer Auswärtiger Dienst: Strategy for Security and Development in the Sahel, S. 1.

43 Ebenda, S. 9.

44 Europäischer Auswärtiger Dienst: Factsheet. The European Union and the Sahel, Brüssel, 14.11.2012, S. 1.

45 Beschluss 2012/392/GASP des Rates vom 16. Juli 2012 über die GSVP-Mission der Europäischen Union in Niger (EUCAP Sahel Niger), in: Amtsblatt der EU, Nr. L 187 vom 17. Juli 2013, S. 48-51.

46 Das Projekt wurde nach dem Putsch in Mali gestoppt.

47 Zeit Online: Die Bundeswehr ist längst in Mali, 29.10.2012.

48 Allein 6.000 Franzosen leben in Mali.

49 Niger ist Frankreichs Hauptlieferant für Uran.

50 Durch die Sahara läuft eine der Hauptschmuggelrouten für Kokain. 
die Bildung einer Übergangsregierung, bestand aber auf einer ,glaubwürdigen und konsensualen roadmap“ ${ }^{51}$ Brüssel erklärte sich bereit, Mali mit einem entwicklungs- und sicherheitspolitische Komponenten umfassenden Ansatz zu unterstützen. Dazu gehören ein verstärktes Engagement in der Sahelzone, der Einsatz der „European Union military mission to contribute to the training of the Malian Armed Forces“ (EUTM Mali) und - nach der Eskalation des Konflikts - die bilaterale Unterstützung des französischen Militäreinsatzes.

,Brüssel' verfolgt mit der EUTM Mali das strategische Ziel, die Fähigkeiten der malischen Armee zu verbessern, um sie zu befähigen, ein sicheres Umfeld für den Transitionsprozess zu schaffen, die territoriale Integrität des Staates wiederherzustellen und den Aufbau einer rechtsstaatlichen Ordnung im Norden zu fördern. Konkret soll EUTM Mali das Land in die Lage versetzen, Kampfoperationen durchzuführen, wobei eine eigene Beteiligung daran explizit ausgeschlossen wird. Die EU-Mission strebt dreierlei an: Ausbildung von vier Einsatzgruppen in Bataillonsstärke in militärischem Know-how sowie Menschenrechten, internationalem humanitärem Recht und Schutz der Zivilbevölkerung; Beratung zum Aufbau einer funktionierenden Befehlskette; Unterstützung der logistischen Fähigkeiten. Mittelfristig will man auch strukturelle Probleme, zum Beispiel beim Personalmanagement angehen. Die Mission ist bis zum Frühjahr 2014 mandatiert. ${ }^{52}$

Gleichzeitig unterstützen Soldaten der ECOWAS mit der „African-led International Support Mission in Mali“ (AFISMA) die malische Armee dabei, Aufständische zu bekämpfen. Die im Dezember 2012 erteilte prinzipielle Erlaubnis der Vereinten Nationen für einen Militäreinsatz der ECOWAS fiel zunächst zögerlich aus, weil der Bericht des Generalsekretärs noch ungelöste Probleme benennt, an erster Stelle die Notwendigkeit eines inklusiven politischen Dialogs der malischen Konfliktparteien mit dem Ziel eines gemeinsames Fahrplans für den politischen Übergang und für die Berücksichtigung der lange bestehenden Klagen der Tuareg und anderer Volksgruppen im Norden. Eine Militäroperation kam für ihn erst als letzte Möglichkeit infrage. Zudem befürchtete er im Falle einer nicht gut geplanten Militäroperation eine Verschlechterung der humanitären Lage, beträchtliche Menschenrechtsverletzungen und das Risiko, jegliche Chancen auf eine Verhandlungslösung zu verspielen. ${ }^{53}$

Doch angesichts des Vormarsches radikalislamistischer Gruppen in Richtung Süden sprach sich der Sicherheitsrat der Vereinten Nationen am 11. Januar 2013 für eine rasche Stationierung der AFISMA aus. ${ }^{54}$ Frankreich startete gleichzeitig auf Bitte der malischen Übergangsregierung die „Operation Serval“. Sie sollte zunächst zusammen mit der malischen Armee den Vormarsch der Aufständischen stoppen. Ob diese wirklich nach Bamako vordringen wollten, ist allerdings unklar. Jedenfalls hätten sie sich im Falle der Einnahme der strategisch wichtigen Stadt Mopti und des nahe gelegenen Flughafens eine strategisch günstige Lage vor Beginn der Regenzeit geschaffen. Für den französischen Präsidenten François Hollande, der zuvor erklärt hatte, dass er keine Bodentruppen einsetzen werde, ${ }^{55}$ handelt es sich um die Abwehr einer „klaren Aggression“ und einer Bedrohung der Existenz Malis. ${ }^{56}$ Also setzte er 4.000 Soldaten einschließlich Panzern, Spezialkräften, Kampfhub-

51 Rat der Europäischen Union: 3199. Tagung des Rates. Auswärtige Angelegenheiten, Dok. 16062/12, S. 9.

52 Europäischer Auswärtiger Dienst: EU Training Mission in Mali launched (EUTM Mali), Brüssel, 13.1.2013.

53 Sicherheitsrat der Vereinten Nationen: Report of the Secretary General on the situation in Mali, S/2012/894, S. 18 und 20.

54 UN News Centre: Mali: UN Security Council expresses 'grave concern' over reports of rebel military activity, abrufbar unter: http://www.un.org/apps/news/story.asp?NewsID=43902\# (letzter Zugriff: 7.2.2013).

55 Olivier Zajec: Vor und nach Timbuktu, in: Le Monde diplomatique, 8.2.2013, S. 10.

56 Süddeutsche Zeitung: Hilfe, Frankreich!, 12./13.1.2012, S. 10. 
schraubern und Kampfflugzeugen ein, die auch Schläge gegen Rebelleneinrichtungen tief im Lande ausführten und die Speerspitze bei der Rückeroberung des Nordens waren.

Frankreich erhielt breite internationale Unterstützung. Es trug zwar die Hauptlast des Militäreinsatzes, wurde allerdings von den europäischen und amerikanischen Verbündeten logistisch und in der Aufklärung unterstützt. ${ }^{57}$ Die Europäische Union passte ihr Krisenmanagementkonzept für die EUTM Mali an: Die Gesamtstärke wurde auf 500 erhöht, die Schutzkomponente verstärkt und das ,gemeinsame Budget“ von sechs auf zwölf Millionen Euro verdoppelt. ${ }^{58}$ Die Hauptkosten von 90 Millionen Euro tragen die teilnehmenden Staaten. Die ECOWAS-Mitglieder sagten die Entsendung von bis zu 6.000 Soldaten zu. Der Tschad entsandte 2.000 Soldaten. Sogar das ursprünglich interventionskritische Algerien öffnete seinen Luftraum und schloss die Grenzen zum Norden Malis. Die Eskalation seitens der Aufständischen verschärfte die humanitäre Lage, zehntausende flohen vor den Kämpfen.

Mit der vorübergehenden Vertreibung der radikalislamistischen Kräfte aus den Städten des Nordens ist ein wichtiges Ziel erreicht worden. Dies gelang durch eine Militäraktion, auf die Paris bereits früh in den Vereinten Nationen gedrängt hatte, aber ohne erfolgreich zu sein. Ein Einsatz der EU-Battle Group, die im ersten Halbjahr 2013 von Deutschland, Frankreich und Polen gestellt wird, wurde zwar im EAD erwogen, aber wegen Unstimmigkeiten und zu hohem Zeitdruck verworfen. ${ }^{59}$ Also führte es die entscheidenden Operationen in nationaler Regie durch, gab sich aber große Mühe, der Aktion ein afrikanisches Gesicht zu geben. Zugleich machte Paris klar, dass die AFISMA schnell übernehmen soll, denn es habe nicht die Absicht, so Premierminister Laurent Fabius, ,getting stuck in the mud“" ${ }^{60}$ Damit deutete er an, dass das Ziel der Ausschaltung der terroristischen Gruppen und der organisierten Kriminalität noch lange nicht erreicht ist. Ob die AFISMA, die später in eine UN-Blauhelmmission überführt werden soll, dazu fähig sein wird, ist fraglich. ${ }^{61}$ Die nun folgende problematische Etappe der Stabilisierung des Staates, die auch Elemente der Aufstandsbekämpfung enthalten wird, ist eine langfristige Aufgabe, welche über die EUTM Mali hinausweist. Sie verlangt nach einer inklusiven Lösung des politischen Konflikts unter Einschluss der Tuareg und anderer Volksgruppen. ${ }^{62}$ Das malische Parlament hat zwar am 30. Januar 2013 einen Fahrplan für die politische Transition einschließlich eines Wahltermins im Sommer verabschiedet, ${ }^{63}$ doch es bleibt abzuwarten, ob er von allen Konfliktparteien akzeptiert wird und ob bis dahin reguläre Wahlen überall gewährleistet werden können. Es besteht also das Ri-

57 Die Kosten für die „Operation Serval“ beliefen sich nach vier Monaten auf circa 200 Millionen Euro. Die Kosten für die Unterstützung durch die Partner werden für den ersten Monat auf 60 bis 100 Millionen Euro geschätzt. Vgl. Sénat: Rapport d’information par le groupe travail „Sahel“, №513 vom 16.4.2013, S. 66; Nicolas GrosVerheyde: Les alliés au Mali : encombrants, inutiles, utiles ? Le vrai, le faux ..., in: Bruxelles2.eu, 14.2.2013.

58 Die ,gemeinsamen Kosten ' betragen nur einen kleinen Teil der insgesamt anfallenden Kosten einer Militäroperation und werden nach dem Athena-Mechanismus berechnet. Vgl. Europäischer Auswärtiger Dienst: Athena, abrufbar unter: https://www.consilium.europa.eu/eeas/security-defence/csdp-structures-and-instrumen ts/financing-of-csdp-military-operations?lang=de (letzter Zugriff: 13.5.2013).

59 Vgl. Nicolas Gros-Verheyde: Les vertus du battlegroup, in: Bruxelles2.eu, 6.2.2013.

60 International Herald Tribune: Timbuktu taken, France wants transition, 30.1.2013, S. 4.

61 Am 25. April 2013 beschloss der Sicherheitsrat der Vereinten Nationen die Aufstellung der United Nations Stabilization Mission in Mali (MINUSMA). Diese circa 13 Soldaten und Polizisten umfassende Stabilisierungstruppe wird unterstützt durch eine französische Eingreiftruppe. Vgl. Vereinte Nationen: Resolution 2100 (2013). Adopted by the Security Council at its $6952^{\text {nd }}$ meeting, on 25 April 2013, S/RES/2100 (2013).

62 Vgl. Barbara Rocksloh-Papendieck/Henner Papendieck: Die Krise im Norden Malis. Aktuelle Lage, Ursachen, Akteure und politische Optionen, Studie der Friedrich-Ebert-Stiftung, Dezember 2012.

63 MaliJet: Assemblée nationale: La feuille de route de la transition fait l'unanimite, 3.11.2012, abrufbar unter: http://www.malijet.com/actualite-politique-au-mali/63314-assembl\%C3\%A9e-nationale-\%3A-la-feuille-de-r oute-de-la-transition-fait-.html?print (letzter Zugriff: 14.2.2013). 
siko, dass mit einer überhasteten oder zu wenig inklusiven Herangehensweise die Saat für neue Konflikte gelegt wird. Zudem muss die regionale Dimension des Problems angegangen werden. Die Europäische Union sollte ihre Sahel-Strategie ausbauen - so müssten Nigeria und Algerien als die beiden an den Sahel angrenzenden politischen Schwergewichte in die EU-Strategie eingebunden werden ${ }^{64}$ - und die afrikanischen Staaten beim Aufbau einer afrikanischen Sicherheitsstruktur stärker unterstützen. ${ }^{65}$

Die Mission der EUTM Mali ist ebenfalls mit Risken behaftet. So könnte der Bewegungsfreiraum des EUTM-Personals durch terroristische Aktivitäten beeinträchtigt werden. Zudem stellt die Präsenz mehrerer Sicherheitsakteure - EUTM Mali, ECOWAS, Mali, Frankreich, Tschad - große Herausforderungen an die Koordinierungsfähigkeiten der Akteure. Das malische Militär ist unzuverlässig und die ethnische Vielfalt in Mali groß, sodass die Gefahr besteht, dass von der Europäischen Union trainierte Soldaten sich absetzen, Einheiten sich auflösen oder gar die Seiten wechseln. ${ }^{66} \mathrm{Zu}$ befürchten ist auch, dass malische Sicherheitskräfte sich menschenrechtswidriger Delikte schuldig machen könnten. ${ }^{67}$ Es stellt sich zudem die Frage, welche malischen Streitkräfte überhaupt ausgebildet werden sollen: die bereits im Einsatz befindlichen oder neu zu rekrutierende? Wenn aber - was eigentlich notwendig ist - eine völlig neue Armee aufgebaut werden soll, dann dürften die für die EUTM Mali veranschlagten 15 Monate bei Weitem nicht ausreichen.

Die Strategie zur erfolgreichen Beendigung der EUTM Mali hängt letztlich davon ab, ob die malische Armee zur Übernahme der Ausbildungsmission in der Lage ist und wie sich der politische Prozess entwickelt. Läuft beides positiv, so könnte die Europäische Union Folgemaßnahmen starten, etwa eine Beratungsmission, welche die Umsetzung der Militärreformen sicherstellt, eine Ausdehnung von EUCAP Sahel Niger nach Mali und in andere Staaten oder die weitere finanzielle und technische Unterstützung für die afrikanischen Truppensteller. Gelingt die politische Transition Malis und die Reform der malischen Sicherheitskräfte nicht, so stünde nicht nur Frankreich, sondern auch die Europäische Union vor der Frage, wie sie mit dem daraus resultierenden Sicherheitsproblem angesichts des amerikanischen Desinteresses an einer direkten Verwicklung in Afrika weiter umgehen solle. ${ }^{68}$

\section{Klaffende Lücke zwischen Anspruch und Wirklichkeit}

Dieser Beitrag hatte das Ziel, die Entwicklung der GASP/GSVP nach dem Inkrafttreten des Vertrags von Lissabon anhand der Aktivitäten der Europäischen Union als Krisenmanager in Somalia, Syrien und Mali zu analysieren. Dieser Praxistest hat gezeigt, dass es sich dabei einerseits um einen schwierigen und langsamen Prozess handelt, andererseits aber Fortschritte erkennbar sind. Anspruch und Wirklichkeit klaffen aber noch weit auseinander. Das durch den Vertrag von Lissabon im Primärrecht verankerte Ziel einer gemeinsamen

64 Vgl. Oladiran Bello: Quick fix or quicksand? Implementing the EU Sahel Strategy, Undación Para Las Relaciones Internacionales Y El Dialogo Exterior: FRIDE Working Paper, November 2012, S. 11-15.

65 Vgl. Nicoletta Pirozzi (Hrsg.): Strengthening the Africa-EU Partnership on Peace and Security. How to Engage African Regional Organizations, Rom 2012.

66 Die USA haben in den letzten vier Jahren über eine halbe Milliarde US-Dollar für den Antiterrorkampf in der Subsahara ausgegeben, auch für die Ausbildung malischer Sicherheitskräfte. Ein großer Teil der von den USA ausgebildeten Tuareg-Eliteeinheiten lief zu den Aufständischen über, die restliche Armee putschte, einschließlich der von ,Washington“ ausgebildeten Kräfte. Vgl. Adam Nossiter/Eric Schmitt/Mark Mazzetti: French strikes in Mali supplant caution of U.S., in: New York Times, 13.1.2013.

67 Afua Hirsch: Mali's army suspected of abuses and unlawfull killings as war rages, in: The Guardian, 19.1.2013.

68 David E. Sanger/Eric Schmitt: U.S. weighs costs of helping in Mali, in: International Herald Tribune, 28.1.2013. 
Verteidigungspolitik bedarf eigentlich ,nur ${ }^{6}$ eines Beschlusses des Europäischen Rates, macht aber wenig Sinn, solange es noch keine stärker integrierte GASP und eine daraus abgeleitete Sicherheitsstrategie gibt. Die Chancen dafür müssten eigentlich in dem Maße steigen, wie sich die nationalen Handlungsmöglichkeiten der Mitgliedstaaten einengen und sich die normativen Grundvorstellungen annähern. Die Erfahrungen aus den hier betrachteten drei Krisen zeigen jedoch, dass die Europäische Union noch weit davon entfernt ist.

Der viel beschworene ,comprehensive approach“ ist mehr Programmatik als Realität. In Syrien hat er noch keine Chance, in Mali steht er nur auf dem Papier. Ein umfassendes Instrumentarium wird nur im Falle Somalias eingesetzt, der verschiedene ,policies` umfassende Ansatz bleibt aber bloß additiv. Selbst die Führung der mittlerweile drei GSVP-Operationen in der Region erfolgt nicht wirklich aus einer Hand. Einerseits können die Aktivierung des operativen Hauptquartiers und seine koordinierende Funktion als Fortschritt gewertet werden; andererseits mangelt es aufgrund der britischen Blockade immer noch an einem ständigen integrierten zivil-militärischen Hauptquartier. Im Falle Malis existiert zwar eine Strategie für die Sahel-Region, die sicherheits- und entwicklungspolitische Ansätze verbindet. Sie greift jedoch sowohl geografisch als auch sektoral nicht weit genug.

Die Schwelle für einen direkten robusten Militäreinsatz im Rahmen der Europäischen Union liegt sehr hoch. Das heißt aber nicht, dass einzelne Mitgliedstaaten nicht dazu bereit sind - allerdings nur kurzzeitig und unter nationaler (Mali) oder unter NATO-Führung (Libyen). Im Falle Somalias handelt es sich um eine polizeiartige maritime Operation und die Unterstützung direkter, robuster Aktionen der Afrikanischen Union gemäß eines Stellvertreteransatzes, der darin besteht, die jeweiligen Partner vor Ort für einen robusten Einsatz zu finanzieren, auszubilden und auszurüsten. Gleiches ist für Mali geplant. Insgesamt scheint sich folgendes Muster herauszukristallisieren: ,Paris‘ und/oder ,London` gehen mit verdecktem oder offenem Einsatz militärischer Mittel voran und beeinflussen so die Agenda, während die anderen EU-Staaten mehr oder weniger folgen. Beide versuchen ,von vorne“ zu führen und die Krise in ihrem Sinne zu beeinflussen, stützen sich dabei aber auf die USA, die wiederum nur unterstützend agieren (,leading from behind '). So setzen ,Paris‘ und ,London' frühzeitig Spezialkräfte ein: In Libyen waren sie die treibenden Kräfte für die Durchführung der Luftangriffe, in Somalia betrieben sie Aufklärung und verdeckte Operationen, in Syrien unterstützten sie die oppositionellen Kräfte, in Mali waren sie die Speerspitze der Militäroperation.

Über den Einsatz von Spezialkräften hinausgehende Operationen finden schnell ihre Grenzen an den mangelnden militärischen Fähigkeiten. So zeigte die Intervention in Libyen, dass ,Paris“ und ,London “ ohne die USA und die NATO in einem Konflikt mittlerer Intensität nicht lange durchhaltefähig sind. ${ }^{69}$ Darum suchen sie wenn immer möglich den Schulterschluss mit, Washington ${ }^{70}$ Diese Erkenntnis gilt auch für einen Konflikt niedriger Intensität wie in Mali, zumal wenn er sich länger hinziehen sollte. Die Frage ist angesichts der sich abzeichnenden Obama-Doktrin - vor allem ,nation-building at home“ und weniger internationale militärische Verwicklungen ${ }^{71}$ - allerdings, ob, inwieweit und unter welchen Bedin-

69 Vgl. Royal United Services Institute: Accidental Heros. Britain, France and the Libya operation, RUSI Interim Campaign Report, September 2011.

70 Die Rückkehr Frankreichs in die militärische Integration der NATO und sein starkes Engagement im Antiterrorkampf in Afrika hat diesen Prozess sicherlich erleichtert. Vgl. Hans-Georg Ehrhart: Homeward bound,... wherever: France's return in the military integration of NATO from a German point of view, in: European Security $1 / 2010$, S. 97-112.

71 Lexington: The Obama doctrine, in: The Economist,1.12.2012. 
gungen ,Washington ' bereit ist, , actor of last resort' zu sein, und was daraus für die GASP/ GSVP folgt. ${ }^{72}$ Des Weiteren ist zu fragen, was die EU-Staaten machen, wenn ihr in allen drei Konfliktgebieten praktizierter Stellvertreteransatz nicht aufgehen sollte.

Wenn man davon ausgeht, dass sich an deren militärischer Leistungsfähigkeit mittelfristig nichts Grundsätzliches ändern wird - nicht zuletzt weil die finanziellen Mittel fehlen und weil Deutschland seine Kultur der militärischen Zurückhaltung nicht aufgibt -, so ergeben sich daraus drei Erfordernisse: Stärkung der GASP, intensivere Suche nach Synergien im verteidigungspolitischen Bereich und Ausbau des zivilen Kriseninstrumentariums. Der Syrienkonflikt hat gezeigt, dass die Europäische Union diplomatisch und sanktionspolitisch rasch agieren kann, wenn die Lage es erfordert. Er macht aber auch deutlich, wie schnell die Grenzen des Krisenmanagements erreicht sind, wenn der Sicherheitsrat der Vereinten Nationen gespalten ist. Am Sicherheitsrat vorbei zu agieren wäre aber völkerrechtswidrig. Also wird von einzelnen Staaten, über Bande gespielt‘, indem man Kräfte vor Ort unterstützt.

Die von Jürgen Habermas im Rahmen der Debatte über die wirtschafts- und währungspolitische Integration aufgeworfene Alternative - entweder Preisgabe des Euro und irreparable Schädigung der europäischen Einigung oder Vertiefung der politischen Union, sodass Transfers demokratisch legitimiert werden können ${ }^{73}$ - stellt sich im Bereich der Außen- und Sicherheitspolitik so nicht. Die Europäische Union trägt gegenwärtig keine irreparablen Schäden davon, wenn sie diese Integration nicht weiter vertieft. Angesichts der globalen Herausforderungen müsste die GASP/GSVP gleichwohl weiter entwickelt werden.

Der Bericht der Future of Europe Group, der am 17. September 2012 von elf Außenministern vorgelegt wurde, verlangt zudem weitere Reformen. Er schlägt unter anderem vor, die Rolle der HV/VP gegenüber den anderen Kommissaren mit außenpolitischen Kompetenzen weiter zu stärken, einen einheitlichen Rüstungsmarkt zu schaffen, die Präsidenten direkt wählen zu lassen und sogar die Möglichkeit einer europäischen Armee ins Auge zu fassen. ${ }^{74}$ Ein von 14 Außenministern verfasstes Non-Paper zur Reform des EAD vom Februar 2013 plädiert für eine Reform der Krisenmanagementstrukturen, um zu einem ,wirklich zivil-militärischen Ansatz in der GASP zu kommen“. ${ }^{75}$ Dass diese in einem informellen Rahmen erarbeiteten Anregungen überhaupt zustande gekommen sind, zeigt, dass der dringende Reformbedarf erkannt worden ist. Die vielen Einschränkungen und Vorbehalte belegen aber auch, dass es Fortschritte, wenn überhaupt, nur schrittweise geben wird. ${ }^{76}$ Die erfolgten Kürzungen in den Verteidigungsbudgets der Mitgliedstaaten - durchschnittlich 5,7 Prozent in 2010 und 3,7 Prozent in 2011 - und die angesichts der Finanzkrise auch künftig notwendigen Kürzungszwänge ,schreien` förmlich nach mehr sicherheits- und verteidigungspolitischer Integration. Doch solange das ,nationale Hemd' näher ist als der, europäische Rock', wird sich nicht viel ändern.

Das eingangs als realistischste Variante eingestufte Modell des Europas der immer engeren Union der Völker als Gebilde sui generis schließt die Möglichkeit eines Übergangs in

72 George Friedman: Avoiding the Wars that Never End, Geopolitical Weekly, 15.1.2013, S. 3.

73 Jürgen Habermas: Heraus aus dem Teufelskreis, in: Süddeutsche Zeitung, 22.9.2012.

74 Abschlussbericht der Gruppe zur Zukunft Europas der Außenminister Belgiens, Dänemarks, Deutschlands, Frankreichs, Italiens, Luxemburgs, der Niederlande, Österreichs, Polens, Portugals und Spaniens, 17.9.2012.

75 Nicolas Gros-Verheyde: Comment renforcer le SEAE? Une lettre des ,14‘, in: Bruxelles2.eu, 11.2.2013.

76 Darum kritisierte der deutsche Verteidigungsminister Thomas de Maizière auf der Münchner Sicherheitskonferenz am 1. Februar 2013 Visionen wie die einer europäischen Armee und forderte stattdessen die Umsetzung bereits beschlossener Maßnahmen und eine pragmatische Zusammenarbeit zwischen der Europäischen Union und der NATO. Thomas de Maizière: Rede des Ministers Thomas de Maizière auf der 49. Münchner Sicherheitskonferenz, 1.2.2013. 
eine höhere Integrationsstufe ebenso wenig aus wie zeitweiligen Stillstand oder gar eine regressive Entwicklung. Die Forderung nach einer ,europäischen Armee‘ ist angesichts fehlender europäischer Legitimation und nationaler Widerstände auf absehbare Zeit ebenso illusorisch wie eine Rückkehr zum traditionellen Nationalstaat. Bleibt ein differenzierter Ansatz, bei dem, salopp gesagt, einige Mitgliedstaaten vorpreschen und andere Mitgliedstaaten bremsen, aber am Ende alle zusammen das zerbrochene Geschirr aufräumen müssen. Die Frage ist nur, mit welchem Erfolg?

\section{Facetten europäischer Integration}

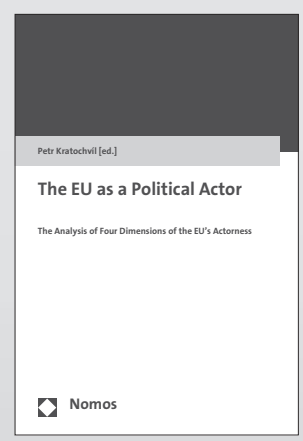

Der Band arbeitet anhand von vier Dimensionen (Anerkennung, Attraktivität, Legitimation und Rahmung) heraus, welche Bedeutung es für die EU hat, ein internationaler Akteur zu
The EU as a Political Actor The Analysis of Four Dimensions of the EU's Actorness

Herausgegeben von

Petr Kratochvíl

2013, 199 S., brosch., 34,- $€$

ISBN 978-3-8329-7223-3

sein. Das internationale Autorenteam versammelt interdisziplinäre Beiträge, die sich aus theoretischer und empirischer Sichtweise mit der Thematik beschäftigen.

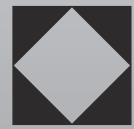

